\title{
Metachronous or synchronous primary lung cancer in the era of computed tomography surveillance
}

\author{
Yuzhao Wang, MD, ${ }^{\text {a,b }}$ Jonathan C. Yeung, MD, PhD, ${ }^{\text {a }}$ Waël C. Hanna, MBA, MD, ${ }^{c}$ Frances Allison, MSc, ${ }^{a}$ \\ Narinder S. Paul, MD, ${ }^{\mathrm{d}}$ Thomas K. Waddell, MD, PhD, ${ }^{\mathrm{a}}$ Marcelo Cypel, MD, ${ }^{\mathrm{a}}$ Marc E. de Perrot, MD, ${ }^{a}$ \\ Kazuhiro Yasufuku, MD, PhD, ${ }^{a}$ Shaf Keshavjee, MSc, MD, ${ }^{a}$ Andrew F. Pierre, MSc, MD, ${ }^{a}$ and \\ Gail E. Darling, MD, FRCSC ${ }^{a}$
}

\section{ABSTRACT}

Objectives: The purpose of this study was to determine the frequency, characteristics, and survival of second primary lung cancer initially identified as an indeterminate lesion on the original computed tomography scan and then diagnosed during the surveillance period in a prospective study.

Methods: A prospective database of 271 patients enrolled in a surveillance study was updated. Indeterminate lesions present on the original computed tomography at the time of initial primary lung cancer diagnosis that subsequently grew and were diagnosed as cancer were termed "synchronous primary lung cancer." Lesions that were not present on the original computed tomography scan and subsequently diagnosed on surveillance were termed "metachronous primary lung cancer."

Results: Thirty patients (11.1\%) developed 37 second primary lung cancers over a median surveillance period of 84.7 (range, 15.9-147.6) months. Of these, 15 of $37(40.5 \%)$ were identified as synchronous primary lung cancer, and 22 of 37 $(59.5 \%)$ were identified as metachronous primary lung cancer. At first identification, ground-glass lesions were identified in 9 of $15(60 \%)$ synchronous primary lung cancers compared with only 5 of $22(22.7 \%)$ of metachronous primary lung cancers $(P=.034)$. Compared with metachronous primary lung cancer, from first identification to diagnosis, synchronous primary lung cancer developed over a longer interval (33.6 vs 7.2 months, $P=.001$ ) and had a slower growth rate ( 0.17 vs $0.45 \mathrm{~mm} / \mathrm{month}, P=.027)$. The 5 -year overall survival from second lung cancer was $73.0 \%$. No significant differences were observed between the synchronous primary lung cancer and metachronous primary lung cancer cohorts in overall survival from initial primary lung cancer $(P=.583)$ or from second lung cancer $(P=.966)$.

Conclusions: Computed tomography surveillance identifies 2 types of curable second lung cancers leading to excellent overall survival. (J Thorac Cardiovasc Surg 2019;157:1196-202)

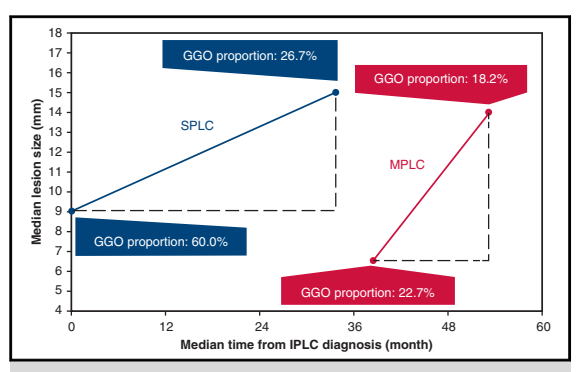

SPLC had a more rapid increase in cancer size than MPLC from identification to diagnosis.

\section{Central Message}

CT surveillance identifies 2 types of curable second lung cancers leading to excellent overall survival.

\section{Perspective}

Lung cancer survivors have a high risk of developing another primary one. We reviewed and updated the database of a prospective study to characterize 2 types of second lung cancers diagnosed during the $\mathrm{CT}$ surveillance period after initial tumor resection. Despite having a different natural course, the overall survival of both was excellent. Surveillance with CT after lung cancer resection is worthwhile in that it allows identification of potentially curable second lung cancers.

\section{See Commentary on page 1203.}

See Editorial page 1194.
From the ${ }^{\text {a Division }}$ of Thoracic Surgery, Department of Surgery, Toronto General Hospital, University of Toronto, Toronto, Ontario, Canada; ${ }^{b}$ Key Laboratory of Carcinogenesis and Translational Research (Ministry of Education), Department of Thoracic Surgery, Peking University Cancer Hospital \& Institute, Beijing, PR China; ' Division of Thoracic Surgery, McMaster University, Hamilton, Ontario, Canada; and ${ }^{\mathrm{d}}$ Department of Medical Imaging, Western University, London, Ontario, Canada.

Received for publication Feb 18, 2018; revisions received Sept 10, 2018; accepted for publication Sept 13, 2018; available ahead of print Nov 2, 2018

Address for reprints: Gail E. Darling, MD, FRCSC, Division of Thoracic Surgery, Department of Surgery, Toronto General Hospital, University of Toronto, 200 Elizabeth St, 9N-955, Toronto, Ontario M5G 2C4, Canada (E-mail: gail.darling@uhn. ca).

0022-5223/\$36.00

Copyright (c) 2018 by The American Association for Thoracic Surgery

https://doi.org/10.1016/j.jtcvs.2018.09.052
With advances in both surgical and medical therapy for lung cancer, and with the prevalence of computed tomography (CT) imaging for screening, an increasing number of patients with early-stage lung cancer are identified and surviving. ${ }^{1}$ However, these patients are still among the highestrisk population for developing another primary lung cancer. Among them, the incidence of developing a new primary lung cancer that is diagnosed on surveillance is 4 to 6 times higher than the baseline population, and the median 10-year risk is $8.36 \%$ (range, $0.56-14.3$ ). ${ }^{1,2}$ Given the high risk of lung cancer in such patients, there is some rationale for following them with $\mathrm{CT}$ whether under the guise of 


\section{Abbreviations and Acronyms \\ $\mathrm{AAH}=$ atypical adenomatous hyperplasia \\ CI = confidence interval \\ $\mathrm{CT}=$ computed tomography \\ IPLC = initial primary lung cancer \\ MnDCT $=$ minimum-dose computed tomography \\ MPLC = metachronous primary lung cancer \\ SPLC = synchronous primary lung cancer}

surveillance or lung cancer screening. Thus, close longterm follow-up after curative surgical resection of lung cancer is recommended. ${ }^{3}$ There is little high-level evidence supporting surveillance for lung cancer survivors; however, published data have demonstrated that surveillance with minimum-dose computed tomography (MnDCT) of the chest is superior to chest $\mathrm{x}$-ray for the detection of a new or recurrent pulmonary lesion. ${ }^{3-5}$ Surveillance with CT leads to earlier detection and a higher rate of curative treatment of second lung cancer at an asymptomatic phase, so prolonged survival may be achievable. ${ }^{4,6} \mathrm{~A}$ systematic review and meta-analysis identified that asymptomatic recurrence was associated with increased survival (hazard ratio, 0.61, $P<.01$ ); thus, it appears that despite some negative studies, the summation of the available evidence supports that there is likely a patient population who may benefit from CT surveillance. This survival benefit was not seen in symptomatic patients, again suggesting the benefit of early identification of recurrence.

During our previous study of surveillance with $\mathrm{MnDCT},{ }^{4}$ we occasionally identified that a second primary lung cancer detected by CT surveillance was present at the same location in the lung on the original CT that identified the first primary lung cancer, but was considered indeterminate. As such, those tumors should be considered synchronous primary lung cancers (SPLCs). In the present study, we reviewed and updated the database of our previous study to determine the frequency of this type of second lung cancer in our enrolled cohort. We also characterized the clinical and imaging patterns of this second lung cancer and its impact on long-term outcome of patients.

\section{PATIENTS AND METHODS}

The research ethics board of University Health Network approved this study. Patient consent had been obtained when conducting the previous MnDCT study. The prospective database of the MnDCT study enrolled 311 patients who underwent lung cancer resection followed by surveillance with MnDCT per protocol at Toronto General Hospital between 2005 and 2012. ${ }^{4}$ All patients met the entry criteria for the previous study and were assessed in similar fashion. After excluding those who withdrew from the study or failed to be followed up, 271 were reviewed and analyzed in the present study. The first resected primary lung tumor for which patients were enrolled into the surveillance protocol was defined as the initial primary lung cancer (IPLC). The primary lung tumors diagnosed during surveillance period were defined as second lung cancer. Patients in the database developing second lung cancer were selected as the subjects of the present study. The diagnosis of second lung cancer was determined by histomorphology or molecular testing. The criteria of Martini and Melamed were followed when similar histology was observed between IPLC and second lung cancer: Distinct pulmonary malignancy is considered a second primary cancer if it fulfills any 1 of the following 3 conditions: (1) a disease-free interval between the 2 tumors of at least 2 years; (2) development of the new tumor from carcinoma in situ; (3) occurrence of the second tumor in a different lobe or lung without positive intervening lymph nodes and evidence of extrapulmonary metastasis. Recommendations of the multidisciplinary tumor board were also important when diagnosing difficult cases. Patients with lack of histologic/cytologic confirmation of second lung cancer were excluded from the study.

In the patients who developed a second lung cancer, images and reports of chest CT scans during the surveillance period were reviewed, which included all the scans from IPLC diagnosis to second lung cancer diagnosis. Second lung cancers that were found to have been present as indeterminant lesions at the same location in the lung on the original $\mathrm{CT}$ at the time of the IPLC diagnosis were defined as SPLC. Second lung cancers that were not present on the original $\mathrm{CT}$ scan were defined as metachronous primary lung cancer (MPLC). Therefore, for SPLC, the time of the first identification of the lesion was considered as the same time of IPLC diagnosis. In contrast, the time of the first identification of MPLC was determined by CT image review.

Imaging density of lesions was classified as ground-glass, solid, partsolid (containing both ground-glass region and solid region), and cavitary. Lesion size on imaging was defined as the diameter in the longest axis of lesion. Growth rate was defined as the average growth rate of size per month during surveillance period, which was calculated by the following algorithm: (size at diagnosis - size at first identification)/interval months from first identification to diagnosis. IPLC, SPLC, and MPLC were restaged according to the eighth edition of the TNM classification.

The follow-up data of the previous study were updated to August 2017. The surveillance protocol had also continued until that time, with MnDCT scans at 3, 6, 12, 18, and 24 months after IPLC surgery and then 1 scan annually if patients had no evidence of disease. ${ }^{4}$ Patients whose last lung cancer was SPLC were analyzed in the SPLC cohort; those whose last lung cancer was MPLC were analyzed in the MPLC cohort. IPLC survival time was calculated from the date of the diagnosis of IPLC to the date of death or last follow-up. Second lung cancer survival time was calculated from the date of the diagnosis of the last second lung cancer to the date of death or last follow-up. Categoric variables were compared by the chisquare test or the Fisher exact test. Continuous variables were compared by the Student $t$ test or the Mann-Whitney $U$ test. Overall survival was calculated by the Kaplan-Meier method, and differences were assessed using log-rank analysis. All statistical analyses were conducted with SPSS 21.0 software (SPSS Inc, Chicago, Ill).

\section{RESULTS}

Of 271 patients in the database, $30(11.1 \%)$ developed 37 second lung cancers during the surveillance period. The baseline and IPLC characteristics of the 30 patients are listed in Table 1 . Three patients $(3 / 30,10.0 \%)$ had multiple synchronous IPLCs that were resected in the same specimen and had the same histology and stage for each patient. Most patients underwent lobectomies for IPLC (21/ $30,70.0 \%)$ and had stage I disease $(26 / 30,86.7 \%)$. Adenocarcinoma was diagnosed in 24 patients $(80.0 \%)$. Three 
TABLE 1. Baseline and initial primary lung cancer characteristics of patients

\begin{tabular}{|c|c|}
\hline Characteristics & $\mathbf{N}=\mathbf{3 0}$ \\
\hline Age at IPLC, median (range), y & $68.9(51.8-90.2)$ \\
\hline \multicolumn{2}{|l|}{ Gender, No. $(\%)$} \\
\hline Male & $16(53.3)$ \\
\hline Female & $14(46.7)$ \\
\hline \multicolumn{2}{|l|}{ Smoking history, No. (\%) } \\
\hline Yes & $23(76.7)$ \\
\hline No & $7(23.3)$ \\
\hline \multicolumn{2}{|l|}{ Histology, No. (\%) } \\
\hline Adenocarcinoma & $24(80.0)$ \\
\hline Squamous cell carcinoma & $6(20.0)$ \\
\hline \multicolumn{2}{|l|}{ Number of synchronous lesions, No. (\%) } \\
\hline 1 & $27(90.0)$ \\
\hline 2 & $2(6.7)$ \\
\hline 3 & $1(3.3)$ \\
\hline \multicolumn{2}{|l|}{ Pathological stage, No. (\%) } \\
\hline I & $26(86.7)$ \\
\hline II & $3(10.0)$ \\
\hline III & $1(3.3)$ \\
\hline \multicolumn{2}{|l|}{ Extent of lung resection, No. (\%) } \\
\hline Lobectomy & $21(70.0)$ \\
\hline Segmentectomy & $4(13.3)$ \\
\hline Wedge & $4(13.3)$ \\
\hline Bilobectomy & $1(3.3)$ \\
\hline \multicolumn{2}{|l|}{ Induction therapy, No. (\%) } \\
\hline Yes & $1(3.3)$ \\
\hline No & $29(96.7)$ \\
\hline \multicolumn{2}{|l|}{ Adjuvant chemotherapy, No. (\%) } \\
\hline Yes & $3(10.0)$ \\
\hline No & $27(90.0)$ \\
\hline Length of follow-up, median (range), mo & $84.7(15.9-147.6)$ \\
\hline
\end{tabular}

$I P L C$, Initial primary lung cancer.

patients $(3 / 30,10.0 \%)$ received adjuvant chemotherapy after the initial resection. The median length of follow-up was 84.7 months (range, 15.9-147.6).

Of 37 second lung cancers, $20(54.1 \%)$ were diagnosed by image-guided biopsy and $17(45.9 \%)$ were diagnosed by surgical resection; $15(40.5 \%)$ were identified as SPLC and $22(59.4 \%)$ were identified as MPLC. Two patients had multiple second lung cancers during the surveillance period. One patient had 3 (1 SPLC and 2 MPLC diagnosed at different times), and one patient had 6 (1 SPLC and 5 MPLC, 4 diagnosed at the same time). Except for 1 SPLC leading to mild shortness of breath, all the second lung cancers were asymptomatic. The characteristics of second lung cancer and the comparison between SPLC and MPLC are shown in Table 2. No significant differences were observed between SPLC and MPLC in characteristics regarding location, histology, stage, and type of therapy.
Ten $(10 / 15,66.7 \%)$ indeterminate lesions of SPLC were reported by radiologists at the time of diagnosis of IPLC. Five of the SPLCs were identified when study authors reviewed the original $\mathrm{CT}$ scans and identified a lesion in the same location as the SPLC but that was not described in the original CT report. The median interval between the diagnosis of IPLC and the first identification of MPLC on CT image was 38.4 months (range, 7.0119.0). There was no significant difference in lesion size between SPLC and MPLC at the time of first identification ( 9.0 vs $6.5 \mathrm{~mm}, P=.566$ ). However, the proportion of ground-glass lesion at first identification of SPLC was higher compared with MPLC $(60 \%$ vs $22.7 \%$, $P=.034)$. The majority of MPLCs $(73 \%)$ were solid at the time of first identification. There was a longer interval from first identification to diagnosis for SPLC lesions compared with MPLC (33.6 vs 7.2 months, $P=.001$ ). At diagnosis, there was no difference in distribution of imaging density between SPLC and MPLC $(P=.300)$, although a higher proportion of SPLC lesions changed imaging density compared with MPLC from first identification to diagnosis $(40 \%$ vs $9.1 \%, P=.042)$. There was no significant difference in the lesion size between SPLC and MPLC at diagnosis (15.0 vs $14.0 \mathrm{~mm}$, $P=.710$ ); however, the growth rate from first identification to diagnosis was slower for SPLC compared with MPLC ( 0.17 vs $0.45 \mathrm{~mm} / \mathrm{month}, P=.027$ ). There was a longer time interval between the diagnosis of IPLC and the diagnosis of MPLC compared with SPLC (53.1 vs 33.6 months, $P=.001$ ) (Table 2). The development of SPLC and MPLC from first identification to diagnosis is summarized in Figure 1.

The 5-year and 10-year IPLC survival for the whole cohort was $86.7 \%$ (95\% confidence interval [CI], 74.598.9) and $79.2 \%$ (95\% CI, 64.3-94.1), respectively. The 5 -year second lung cancer survival was $73.0 \%$ (95\% CI, 52.6-93.4). No significant differences between SPLC and MPLC cohort were observed in the overall survival after IPLC $(P=.583$, Figure 2$)$ or after second lung cancer $(P=.966$, Figure 3$)$.

\section{DISCUSSION}

There are few previous published data exclusively addressing the second primary lung cancer in which an abnormality in the lung on the original CT used to assess the IPLC was subsequently identified as cancer during surveillance. Dai and colleagues ${ }^{9}$ reported 4 of 43 patients whose synchronous ground-glass nodules along with initial lung cancer were diagnosed as second primary tumor during the surveillance period after the initial resection. Gao and colleagues ${ }^{10}$ found that therapeutic interventions were performed because of the progression of these lesions during surveillance in 11 of 70 patients with unresected 
TABLE 2. Characteristics of second lung cancer and comparison between synchronous primary lung cancer and metachronous primary lung cancer

\begin{tabular}{|c|c|c|c|c|}
\hline Characteristics & $\begin{array}{c}\text { Second lung } \\
\text { cancer }(\mathbf{n}=37)\end{array}$ & $\begin{array}{c}\text { SPLC } \\
(\mathbf{n}=\mathbf{1 5})\end{array}$ & $\begin{array}{l}\text { MPLC } \\
(\mathrm{n}=\mathbf{2 2})\end{array}$ & $\begin{array}{l}P \text { value (SPLC } \\
\text { vs MPLC) }\end{array}$ \\
\hline Interval from IPLC to first identification, median (range), mo & N/A & N/A & $38.4(7.0-119.0)$ & N/A \\
\hline Interval from IPLC to diagnosis, median (range), mo & $42.4(5.8-133.3)$ & $33.6(5.8-58.8)$ & $53.1(13.6-133.3)$ & .001 \\
\hline Interval from first identification to diagnosis, median (range), mo & $14.6(0.4-80.1)$ & $33.6(5.8-58.8)$ & $7.2(0.4-80.1)$ & .001 \\
\hline \multicolumn{5}{|l|}{ Location, No. (\%) } \\
\hline Peripheral & 35 (94.6) & $14(93.3)$ & $21(95.5)$ & .779 \\
\hline Central & $2(5.4)$ & $1(6.7)$ & $1(4.5)$ & \\
\hline Ipsilateral to IPLC & $12(32.4)$ & $3(20.0)$ & $9(40.9)$ & .182 \\
\hline Contralateral to IPLC & $25(67.6)$ & $12(80.0)$ & $13(59.1)$ & \\
\hline \multicolumn{5}{|l|}{ Imaging density, No. $(\%)$} \\
\hline At first identification & & & & .034 \\
\hline Solid & $21(56.8)$ & $5(33.3)$ & $16(72.7)$ & \\
\hline Ground-glass & $14(37.8)$ & $9(60.0)$ & $5(22.7)$ & \\
\hline Part-solid & $1(2.7)$ & $1(6.7)$ & $0(0.0)$ & \\
\hline Cavitary & $1(2.7)$ & $0(0.0)$ & $1(4.5)$ & \\
\hline At diagnosis & & & & .300 \\
\hline Solid & $21(56.8)$ & $6(40.0)$ & $15(68.2)$ & \\
\hline Ground-glass & $8(21.6)$ & $4(26.7)$ & $4(18.2)$ & \\
\hline Part-solid & $4(10.8)$ & $3(20.0)$ & $1(4.5)$ & \\
\hline Cavitary & $4(10.8)$ & $2(13.3)$ & $2(9.1)$ & \\
\hline $\begin{array}{l}\text { No change from first identification to diagnosis in imaging } \\
\text { density }\end{array}$ & $29(78.4)$ & $9(60.0)$ & $20(90.9)$ & .042 \\
\hline $\begin{array}{l}\text { Changing from first identification to diagnosis in imaging } \\
\text { density }\end{array}$ & $8(21.6)$ & $6(40.0)$ & $2(9.1)$ & \\
\hline \multicolumn{5}{|l|}{ Lesion size, median (range), $\mathrm{mm}$} \\
\hline At first identification & $7.0(3.0-45.0)$ & $9.0(3.0-18.0)$ & $6.5(3.0-45.0)$ & .566 \\
\hline At diagnosis & $15.0(4.0-45.0)$ & $15.0(10.0-32.0)$ & $14.0(4.0-45.0)$ & .710 \\
\hline Growth rate, median (range), $\mathrm{mm} / \mathrm{mo}$ & $0.24(0.00-4.13)$ & $0.17(0.05-0.94)$ & $0.45(0.00-4.13)$ & .027 \\
\hline \multicolumn{5}{|l|}{ Histology, No, $(\%)$} \\
\hline Adenocarcinoma & $31(83.8)$ & $14(93.3)$ & $17(77.3)$ & .361 \\
\hline Squamous cell carcinoma & $4(10.8)$ & $1(6.7)$ & $3(13.6)$ & \\
\hline Small cell lung cancer & $2(5.4)$ & $0(0.0)$ & $2(9.1)$ & \\
\hline Same as IPLC & $30(81.1)$ & $13(86.7)$ & $17(77.3)$ & .677 \\
\hline Different than IPLC & 7 (18.9) & $2(13.3)$ & $5(22.7)$ & \\
\hline Clinical stage, No. (\%) & & & & .404 \\
\hline I & $31(83.8)$ & $13(86.7)$ & $18(81.8)$ & \\
\hline II & $3(8.1)$ & $1(6.7)$ & $2(9.1)$ & \\
\hline III & $1(2.7)$ & $1(6.7)$ & $0(0.0)$ & \\
\hline IV & $2(5.4)$ & $0(0.0)$ & $2(9.1)$ & \\
\hline Type of therapy, No. (\%) & & & & .513 \\
\hline Wedge resection & $11(29.7)$ & $6(40.0)$ & $5(22.7)$ & \\
\hline Segmentectomy & $4(10.8)$ & $3(20.0)$ & $1(4.5)$ & \\
\hline Lobectomy & $6(16.2)$ & $1(6.7)$ & $5(22.7)$ & \\
\hline SBRT/radiation & $10(27.0)$ & $4(26.7)$ & $6(27.3)$ & \\
\hline Chemotherapy & $2(5.4)$ & $1(6.7)$ & $1(4.5)$ & \\
\hline Chemotherapy + radiation & $1(2.7)$ & $0(0.0)$ & $1(4.5)$ & \\
\hline EGFR-TKI & $1(2.7)$ & $0(0.0)$ & $1(4.5)$ & \\
\hline None & $2(5.4)$ & $0(0.0)$ & $2(9.1)$ & \\
\hline
\end{tabular}

SPLC, Synchronous primary lung cancer; $M P L C$, metachronous primary lung cancer; $I P L C$, initial primary lung cancer; $N / A$, not available; SBRT, stereotactic body radiation therapy; EGFR-TKI, epidermal growth factor receptor tyrosine kinase inhibitor. 


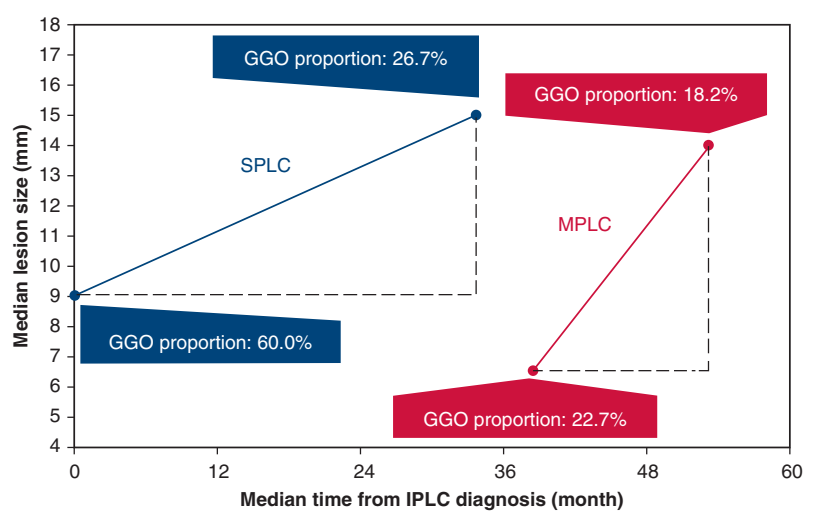

FIGURE 1. Development of size and change of proportion of groundglass opacity from first identification to diagnosis for SPLC and MPLC. The blue curve represents SPLC development, starting at the time of IPLC diagnosis and ending at the median time to SPLC diagnosis (33.6 months). The red curve represents MPLC development, starting at the median time to MPLC first identification (38.4 months) and ending at the median time to its diagnosis (another 7.2 months). The graph depicts the more rapid increase in cancer size of MPLC versus SPLC. It also shows a sharp decrease in ground-glass opacity proportion from first identification to diagnosis in SPLC, but not in MPLC. GGO, Ground-glass opacity; $I P L C$, initial primary lung cancer; $M P L C$, metachronous primary lung cancer; $S P L C$, synchronous primary lung cancer.

synchronous ground-glass nodules identified at the time of initial lung cancer. However, both the 2 studies focused on the management of multifocal ground-grass nodules, which differs from our study. Given the considerable proportion $(15 / 37,40.5 \%)$ of this type of SPLC observed in our data, this scenario is likely frequently encountered in clinical practice. This study evaluates the characteristics and outcome of this particular second lung cancer using an updated database of a prospective study of surveillance using MnDCT scans.

In this study, $60 \%$ of SPLCs at first identification were small ground-glass nodules, which are thought to have a long natural history after a linear multistep progression in which atypical adenomatous hyperplasia (AAH) progresses to adenocarcinoma in situ, which in turn progresses to invasive adenocarcinoma. ${ }^{11}$ There are several articles dealing with the coincidence of AAH and primary lung carcinoma. Suzuki and colleagues ${ }^{12}$ reported synchronous AAH was found in 137 (10\%) of 1360 patients who underwent resection of lung carcinoma in their institution during a period of 24 years. They also reported a special case that was diagnosed as 2 primary adenocarcinomas accompanied with 12 solitary AAH lesions. ${ }^{13}$ Thus, we can speculate that a portion of SPLCs are likely precancerous when identified at the time of IPLC. Although they are termed "synchronous" in our study, SPLCs are actually metachronous for initial cancer in terms of evolution.

Our data identify a higher proportion of ground-glass lesions in SPLC compared with MPLC at the first identification. Moreover, a longer course and a slower growth rate from first identification to diagnosis were observed in SPLC. It appears that SPLC is associated with an indolent nature compared with MPLC.

Our 5-year overall survival from second lung cancer is $73.0 \%$, which is higher than other published rates ranging

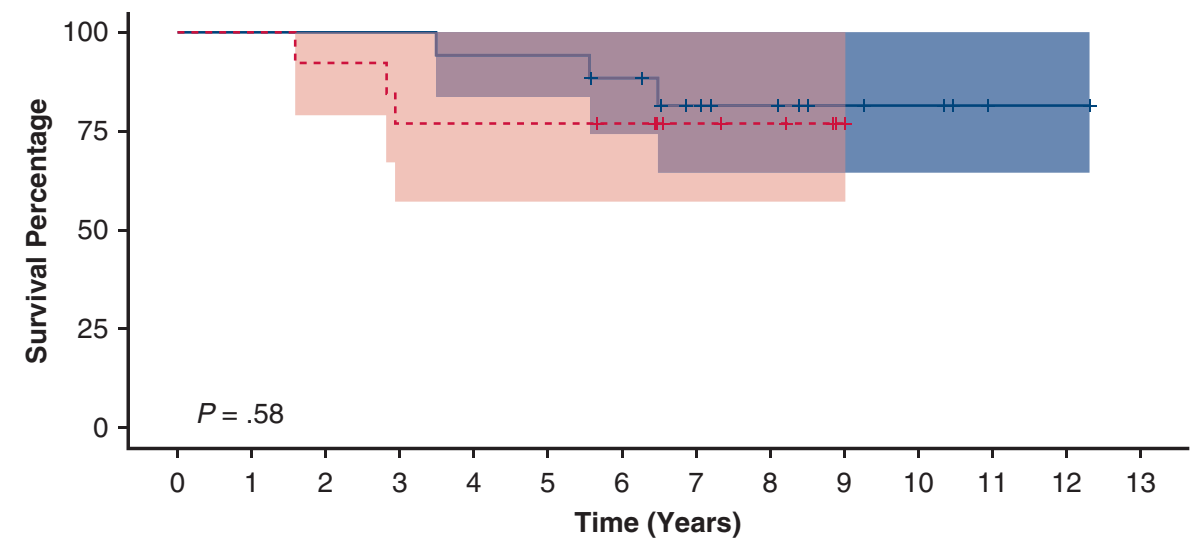

\begin{tabular}{|c|c|c|c|c|c|c|c|c|c|c|c|c|c|c|}
\hline \multirow{4}{*}{$\begin{array}{l}\frac{\pi}{\pi} \text { MPLC - } \\
\text { के SPLC - }\end{array}$} & \multicolumn{14}{|c|}{ Number at risk } \\
\hline & 17 & 17 & 17 & 17 & 16 & 16 & 14 & 10 & 8 & 5 & 4 & 1 & 1 & 0 \\
\hline & 13 & 13 & 12 & 10 & 10 & 10 & 8 & 5 & 4 & 1 & 0 & 0 & 0 & 0 \\
\hline & 0 & 1 & 2 & 3 & 4 & 5 & 6 & 7 & 8 & 9 & 10 & 11 & 12 & 13 \\
\hline & \multicolumn{14}{|c|}{ Time (Years) } \\
\hline
\end{tabular}

FIGURE 2. Comparison of IPLC survival between SPLC and MPLC cohorts. The number of patients at risk is listed under the figure. The colored bands represent the $95 \%$ CIs. No significant differences between the SPLC and MPLC cohorts were observed in overall survival from IPLC. MPLC, Metachronous primary lung cancer; $S P L C$, synchronous primary lung cancer. 


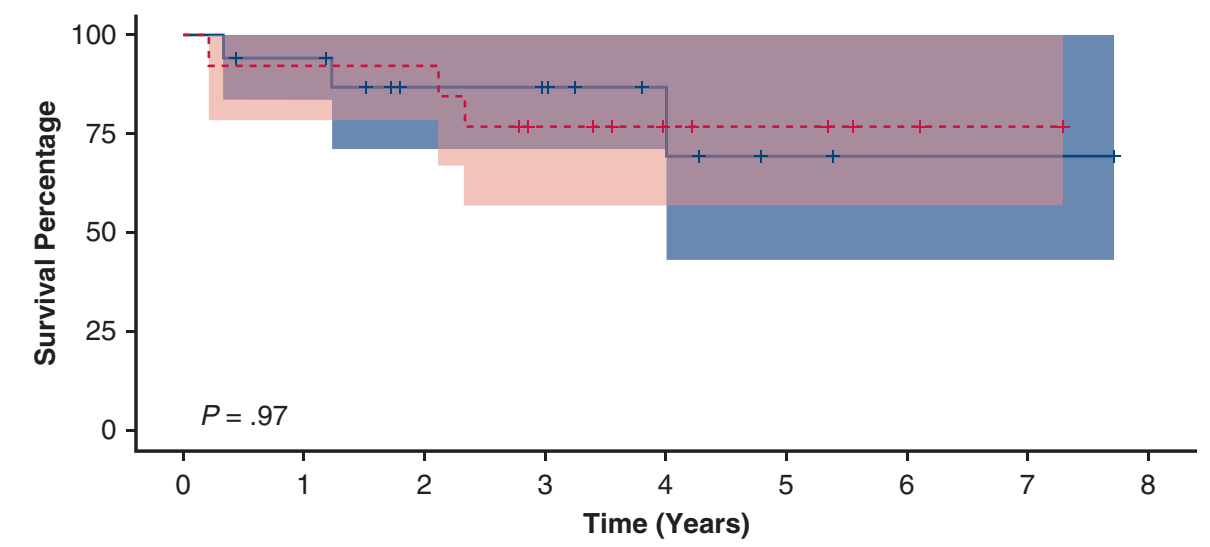

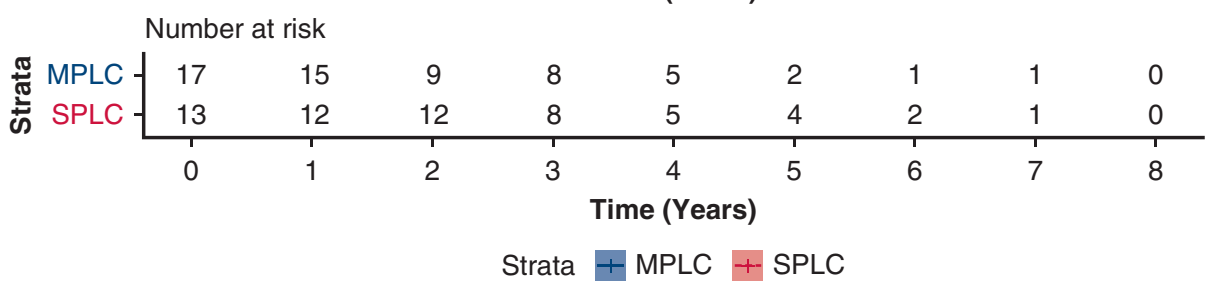

FIGURE 3. Comparison of second lung cancer survival between SPLC and MPLC cohorts. The number of patients at risk is listed under the figure. The colored bands represent the $95 \%$ CIs. No significant differences between the SPLC and MPLC cohorts were observed in overall survival from second lung cancer. $M P L C$, Metachronous primary lung cancer; $S P L C$, synchronous primary lung cancer.

from $20 \%$ to $65 \%,{ }^{14}$ likely a reflection that our original surveillance study included patients with early-stage cancer. Previous studies have reported that patients with a longer interval between IPLC and second cancer had better survival than those with a short interval. ${ }^{14,15}$ In our study, although the MPLC cohort had a longer interval from IPLC to second cancer diagnosis, the survival from IPLC was similar compared with the SPLC cohort. After eliminating this interval, there was no difference in overall survival from second lung cancer between the 2 cohorts.

At the diagnosis of IPLC, the lesions of SPLC were not considered significant and 33.3\% were not even reported by the radiologist reading the initial CT scan. Even when mentioned, these lesions were described as indeterminate because of the tiny volume and nonspecific feature of the lesions compared with IPLC on CT image. Nonetheless, because of the favorable nature of these lesions, 13 of 15 $(86.7 \%)$ SPLCs were stage I at diagnosis. One lesion was stage II (T3N0) because of intralobar metastasis, and the other lesion was stage III (T4N0) because of infiltration of 2 lobes. From the long-term perspective, the survival of SPLC was not inferior to that of MPLC. Therefore, the "neglect" for the lesions of SPLC at the time of IPLC diagnosis seems reasonable.

\section{Study Limitations}

This study has several limitations. This is a singleinstitution experience with limited sample size, so it may not be generalizable to other cohorts. Also, although a prospective database for the study of MnDCT follow-up was used, a small portion of CT images were not available for direct review; thus, we had to determine the detail of those CT scans based on reports only. Moreover, the follow-up CT scans were performed according to the surveillance protocol, but second lung cancer may develop in the interval between CT scans and clinic appointments. All these may lead to data inaccuracies regarding time to diagnosis.

\section{CONCLUSIONS}

A significant proportion of second lung cancers were present at the time of diagnosis of the initial lung cancer but grew slowly, consistent with more indolent biology. Most of these were ground-glass nodules or deemed indeterminate on the initial CT scan. By using a standardized surveillance protocol with low-dose CT chest, these lesions were observed until growth was identified and then were resected. A second population of second lung cancers was also identified by the standardized surveillance protocol. Although this group of metachronous lung cancers grew more quickly and were solid, suggesting a more aggressive biology, they were similarly identified by standardized surveillance at an asymptomatic and curable stage. Despite apparently different biology, survival for both groups was excellent and better than previously reported after resection of second lung cancers. These results suggest that a standardized surveillance protocol using low-dose CT scans of the chest may offer benefit in terms of survival for patients who have undergone resection of early-stage non-small cell lung cancer. 


\section{Conflict of Interest Statement}

Authors have nothing to disclose with regard to commercial support.

\section{References}

1. Surapaneni R, Singh P, Rajagopalan K, Hageboutros A. Stage I lung cancer survivorship: risk of second malignancies and need for individualized care plan. $J$ Thorac Oncol. 2012;7:1252-6.

2. Han SS, Rivera GA, Tammemagi MC, Plevritis SK, Gomez SL, Cheng I, et al. Risk stratification for second primary lung cancer. J Clin Oncol. 2017;35:2893-9.

3. Lou F, Huang J, Sima CS, Dycoco J, Rusch V, Bach PB. Patterns of recurrence and second primary lung cancer in early-stage lung cancer survivors followed with routine computed tomography surveillance. J Thorac Cardiovasc Surg. 2013; 145:75-82.

4. Hanna WC, Paul NS, Darling GE, Moshonov H, Allison F, Waddell TK. Minimal-dose computed tomography is superior to chest x-ray for the follow-up and treatment of patients with resected lung cancer. J Thorac Cardiovasc Surg. 2014; 147:30-3.

5. Hanna WC, Keshavjee S. How to follow up patients after curative resection of lung cancer. Semin Thorac Cardiovasc Surg. 2013;25:213-7.

6. Hamaji M, Allen MS, Cassivi SD, Deschamps C, Nichols FC, Wigle DA Surgical treatment of metachronous second primary lung cancer after complete resection of non-small cell lung cancer. J Thorac Cardiovasc Surg. 2013;145: 683-90.

7. Calman L, Beaver K, Hind D, Lorigan P, Roberts C, Lloyd-Jones M. Survival benefits from follow-up of patients with lung cancer. A systematic review and meta-analysis. J Thorac Oncol. 2011;6:1993-2004.
8. Martini N, Melamed MR. Multiple primary lung cancers. J Thorac Cardiovasc Surg. 1975;70:606-12.

9. Dai C, Ren Y, Xie H, Jiang S, Fei K, Jiang G, et al. Clinical and radiological features of synchronous pure ground-glass nodules observed along with operable non-small cell lung cancer. J Surg Oncol. 2016;113:738-44.

10. Gao RW, Berry MF, Kunder CA, Khuong AA, Wakelee H, Neal JW, et al. Survival and risk factors for progression after resection of the dominant tumor in multifocal, lepidic-type pulmonary adenocarcinoma. J Thorac Cardiovasc Surg. 2017;154:2092-9.

11. Pedersen JH, Saghir Z, Wille MM, Thomsen LH, Skov BG, Ashraf H. Groundglass opacity lung nodules in the era of lung cancer CT screening: radiology, pathology, and clinical management. Oncology (Williston Park). 2016;30:266-74.

12. Suzuki K, Nagai K, Yoshida J, Yokose T, Kodama T, Takahashi K, et al. The prognosis of resected lung carcinoma associated with atypical adenomatous hyperplasia: a comparison of the prognosis of well-differentiated adenocarcinoma associated with atypical adenomatous hyperplasia and intrapulmonary metastasis. Cancer. 1997;79:1521-6.

13. Suzuki K, Takahashi K, Yoshida J, Nishimura M, Yokose T, Nishiwaki Y, et al. Synchronous double primary lung carcinomas associated with multiple atypical adenomatous hyperplasia. Lung Cancer. 1998;19:131-9.

14. Loukeri AA, Kampolis CF, Ntokou A, Tsoukalas G, Syrigos K. Metachronous and synchronous primary lung cancers: diagnostic aspects, surgical treatment, and prognosis. Clin Lung Cancer. 2015;16:15-23.

15. Rosengart TK, Martini N, Ghosn P, Burt M. Multiple primary lung carcinomas: prognosis and treatment. Ann Thorac Surg. 1991;52:773-8.

Key Words: lung cancer, surgery, surveillance, second primary cancer 